\title{
Steuern statt »Durchregieren«
}

\section{Beteiligung dauert, kann aber langfristig besser Wirksamkeit erzielen}

WOLFGANG SCHRANK

Wolfgang Schrank ist Fachbereichsleiter und stellvertretender Geschäftsführer des Frankfurter Vereins für soziale Heimstätten e. V. in Frankfurt am Main. Er ist Mitglied im Beirat der Blätter der Wohlfahrtspflege. wolfgang.schrank@frankfurterverein.de

\author{
Der Wunsch nach schnellen Entschlüssen \\ gefährdet oft wirksame Hilfen. Am Beispiel der \\ Gemeindepsychiatrie in Frankfurt am Main kann \\ gezeigt werden, wie Verständigung und Kooperation \\ zwar unspektakuläre, aber wirksame Angebote \\ entwickeln und dauerhaft sichern können.
}

"Als sie das Ziel aus den Augen verloren hatten, verdoppelten sie ibre Anstrengungen." (Mark Twain)

Das Verhalten der Protagonisten in der Erzählung Mark Twains kommt uns bekannt vor. Entweder sind wir selbst schon wiederholt in diese Falle getreten, beziehungsweise wir haben diese Verhaltensweise an den Tag gelegt. Oder wir meinten, in der Wirtschaft oder Sozialpolitik aufgrund fehlerhafter Steuerung durch andere Akteure beachtliche Fehlentwicklungen vorhersagen oder feststellen zu können. Auch unter ausgewiesenen Fachleuten wird derzeit heftig gestritten, wie und wohin in der Euro- und Finanzkrise gesteuert werden kann und muss - und welche Ziele vernünftigerweise von wem zu erreichen sein müssen.

Entwicklung anzustoßen oder zu beeinflussen ist ein grundlegendes Motiv menschlichen Handelns. Im unternehmerischen Handeln, in privaten Beziehungen, beim Sport und beim Spiel erleben wir das Bedürfnis, durch unser eigenes Handeln den Verlauf ganzer Handlungsketten und langfristiger Abläufe zu beeinflussen.

Wir setzen unsere Kräfte dann wirkungsvoller ein, wenn durch unser gezieltes Handeln berechenbare und vorhersehbare Folgen eintreten. Wir potenzieren diese Ergebnisse, wenn wir in der Lage sind, andere Menschen oder Organisationen produktiv in dieses gezielte Handeln einzubeziehen.

Wenn wir dieses Handeln geplant und gezielt einsetzen, sprechen wir da- von, dass wir einen Prozess oder eine Entwicklung steuern.

Grundsätzlich müssen im Zusammenhang mit einer gezielten Steuerung die folgenden Fragen beantwortet werden:

- Warum soll gesteuert werden, welche Ansprüche bestehen an die Steuerung?

- Wohin soll gesteuert werden?

- Wo und von wem soll gesteuert werden?

- Wann soll gesteuert werden?

- Welche zu steuernden Sachverhalte entfalten eine Wirksamkeit?

Die Steuerung in der Sozialen Arbeit ist dabei zusätzlich mit einer Reihe eigener Bedingungen bzw. Voraussetzungen verbunden:

- So können Ziele in der Regel nicht von einer einzelnen, entschlossen agierenden Person (der »Eigentümer-Persönlichkeit «) alleine definiert werden, sondern sie sind fast immer Ergebnis eines eher langwierigen, demokratischen Aushandlungsprozesses oder einer nicht immer übersichtlichen Entwicklung.

- Die Finanzierung Sozialer Arbeit erfolgt in der Regel aus Budgets, die in irgendeiner Form öffentlich legitimiert oder verwaltet sind. Diese Mittel sind immer sehr begrenzt, »Risikokapital« wird fast nie zur Verfügung gestellt. Dies bedeutet, dass die Steuerung von Prozessen 
oder Maßnahmen zum Erfolg führen müssen - ansonsten wird häufig das erreichte Ergebnis im Nachhinein als das eigentlich angestrebte Ziel ausgegeben.

- Die Steuerung in der Sozialen Arbeit bezieht sich fast immer auf langfristig wirkende Prozesse. Dazu im Widerspruch stehen häufig durch Wahlperioden (sachfremd) definierte Zeitkorridore, Zyklen oder Entwicklungsabschnitte, in die einzelne handelnde Personen eingebunden sind.

- Die angestoßenen Prozesse oder Entwicklungen betreffen reale Menschen in ihrem realen Leben - ausprobieren geht meistens nicht oder wäre inhuman.

- In der Sozialen Arbeit wird häufiger als in der gewerblichen Wirtschaft von den handelnden Personen die Ansicht vertreten, man sei "näher dran « an den Problemen. Zieldefinition und ausführendes Handeln werden so schneller in einen Topf geworfen, das eigene Engagement wird (zu schnell) als Maßstab für die (engagierte) Steuerung genommen.

Drei verschiedene Ebenen der Steuerung sind für einen qualifizierten Lenkungsprozess in der Sozialen Arbeit erforderlich: Neben der Ebene der konkreten Arbeit in der Einzelfallhilfe ist die Ebene der Strukturen, der Ort an dem die Entwicklung der Organisation des Hilfeangebotes stattfindet, eine wichtige Steuerungsinstanz. Als dritte (Steuerungs-) Ebene ist die systematische Kommunikation zur Klärung und Ausrichtung grundlegender Fragen (Ausbau ambulant vor stationär, Stärkung des Betreuten Wohnens zulasten der Entwicklung der Wohnheim-Kapazitäten usw.) zu sehen, die jedoch am wenigsten sichtbar zu konkretem Handeln führt.

Die Abgrenzung dieser drei Ebenen voneinander gelingt nicht immer zur Zufriedenheit aller Beteiligten und auf Dauer - im Bedarfsfall muss nachjustiert werden.

Am Beispiel des Hilfesystems Gemeindepsychiatrie in Frankfurt am Main kann man diese Aufgabenverteilung gut demonstrieren. In der MainMetropole versuchen die verschiedenen Akteure der gemeindepsychiatrischen Versorgung, sich in Fragen der Steuerung dieses Hilfesystems auf diesen drei
Ebenen umfassend zu verständigen. Die Leistungserbringer (vor allem die Verbände der Freien Wohlfahrtspflege), die Betroffenen und ihr soziales Umfeld, die Stadtverwaltung mit ihrem Gesundheits- und Ordnungsamt, die pflichtversorgenden psychiatrischen Kliniken sowie der überörtliche Träger der Sozi- tungen vor Ort erfolgt in halbjährlich stattfindenden Planungskonferenzen. An diesen nehmen der örtliche und der überörtliche Träger der Sozialhilfe sowie die Leistungserbringer teil. Die Federführung dieser Planungskonferenzen liegt beim örtlichen Träger der Sozialhilfe. Die hier getroffenen Fest-

\section{«Ausprobieren kann in der \\ Sozialen Arbeit gefährlich sein - schließlich geht es um reale Menschen in ihrem realen Leben«}

alhilfe als wesentlicher Finanzier dieses Hilfesystems sitzen in unterschiedlichen Gremien zusammen und erörtern, wie sie - nach Möglichkeit weitgehend im Konsens - diese Steuerung wahrnehmen können.

In den zurückliegenden Jahren wurden dafür eine Reihe von Gremien installiert, die mit jeweils abgegrenzter Aufgabe an dieser Steuerung mitwirken sollen:

\section{Erste Ebene: Die Einzelfälle}

Die Steuerung für den Zugang und die Nutzung des Hilfesystems Gemeindepsychiatrie im Einzelfall erfolgt im Rahmen von Hilfeplan- oder Fallkonferenzen. Hier wird in einem Aushandlungsprozess im Detail festgelegt, welche Person in welchem Umfang welche Art der Hilfe erhalten soll. An diesem Aushandlungsprozess sind der örtliche und der überörtliche Träger der Sozialhilfe, die Wohlfahrtsverbände als Leistungserbringer sowie der Betroffene und die von ihm benannten Vertrauenspersonen beteiligt. Die Federführung dieser Hilfeplankonferenzen liegt beim überörtlichen Träger der Sozialhilfe. An diesen richten sich auch die Beschlüsse (d. h. die Empfehlungen) der Hilfeplankonferenzen, denen in der überwiegenden Zahl der Fälle auch entsprochen wird.

\section{Zweite Ebene: Die Strukturen}

Die Steuerung der Entwicklung des Hilfesystems der gemeindepsychiatrischen Versorgung sowie die Entwicklung und Orientierung der Dienste und Einrich- stellungen haben für alle Beteiligten den Charakter von Empfehlungen.

\section{Dritte Ebene: Die Steuerungsgremien}

Die Willensbildung über die Grundzüge der gemeindepsychiatrischen Entwicklung und Hilfen in Frankfurt am Main erfolgt in den Sitzungen des Psychosozialen Ausschusses der Stadt. Dieser tagt zweimal im Jahr und versteht sich in erster Linie als Gremium zur Information und Meinungsbildung. Er trifft in der Regel keine Beschlüsse. Die Federführung des Psychosozialen Ausschusses liegt bei einem Verband der Wohlfahrtspflege.

\section{Verständigung kostet Zeit, garantiert aber Qualität}

Wenn man der hier skizzierten Logik der verschiedenen Entscheidungs- und Steuerungsebenen folgt, kann der einzelne Beschluss einer Dienststelle oder einer politischen Partei kaum direkt einvernehmlich in (sozial-) politisches Handeln umgesetzt werden.

Ein »Durchregieren « ist kaum möglich. Jede neue Idee oder Theorie muss erklärt und vermittelt werden, die jeweils anderen Akteure müssen sich positionieren können und nach Möglichkeit gewonnen werden. Versuche einzelner Akteure in der Vergangenheit, das Hilfesystem durch einseitige Beschlüsse "auf Linie « zu bringen, hatten eher eine Stagnation der Entwicklung als eine Beschleunigung zum Ergebnis.

Dies wird von der Mehrheit der Beteiligten derzeit nicht als Zeichen von 
Verknöcherung oder Unflexibilität des Systems gesehen, sondern als ein Merkmal der komplexen Willensbildung in einer Zivilgesellschaft, die halt nicht immer einfach ist.

\section{Wie sich soziale und betriebswirtschaftliche Steuerung unterscheiden}

Worin unterscheidet sich die Steuerung in der Sozialen Arbeit von der allgemeinen betriebswirtschaftlichen Steuerung?

Privatpersonen und Verbände, Betriebe, politische Gruppen oder sonstige gesellschaftliche Organe definieren ihre Ziele in einem teilweise hoch komplexen Kommunikationsprozess. Eine möglichst breite Beteiligung von (speziell) den betroffenen Menschen und (allgemein) den Bürgerinnen und Bürgern an dieser Zieldefinition ist ausnahmslos von allen politischen Kräften erwünscht.

Diese breite Beteiligung verlangsamt sicher den einen oder anderen Klärungsund Entscheidungsprozess, sie ermöglicht aber auch das "Heranwachsen" von unspektakulären Konzepten und Verfahrensweisen. In der Sozialen Arbeit bedeutet dies, dass sich Debatten und Willensbildungsprozesse über lange Zeiträume hinziehen können, eher nebenbei entschieden werden und meistens nicht zur Profilierung von Einzelpersonen oder Institutionen taugen.

Dafür ist das Ergebnis solcher Verständigungsprozesse normalerweise langfristig wirksam. Dies entspricht nicht immer dem Bedürfnis nach positiver und dynamischer Wahrnehmung der Akteure in der Öffentlichkeit. Auch damit müssen diejenigen leben, die in der Sozialen Arbeit erfolgreich steuern wollen.

In Fragen der Sozialen Arbeit gibt es mindestens so viele Fachleute, wie es Spezialisten für die Mannschaftsaufstellung der Fußball-Nationalmannschaft gibt. Und wenn die lokale Tageszeitung immer (nur) den Volontär über Fragen der Gemeindepsychiatrie schreiben lässt, wird man über einen Zeitraum von drei Jahrzehnten verteilt alle paar Jahre wieder von einem »bislang nie dagewesenen Ansatz für die Arbeit mit Angehörigen« lesen müssen ...

\section{Welche Steuerung dauerhafte Wirksamkeit entfalten kann}

Anscheinend unwichtige Randbedingungen entfalten häufig eine extrem hohe Wirksamkeit, während fachlich eindeutig zuordenbare Entscheidungen, die in spektakulären und öffentlichen Debatten ausgetragen werden, in ihrer Wirksamkeit häufig im Sande verlaufen. So wird vermutlich eine spürbare Anhebung der Schwerbehinderten-Ausgleichsabgabe (für jeden nicht besetzten Platz eines schwerbehinderten Arbeitnehmers) in der gewerblichen Wirtschaft eine deutlich andere Wirkung erzielen, als weitere Diskussionen im Bundestag über die UN-Behindertenrechtskonvention und ihrem Anspruch auf Inklusion und Teilhabe am Arbeitsleben.

Der Glaube an die heilsame Wirkung des Marktes und der Marktkräfte als Lösungshilfe für fast alle Probleme der Gesellschaft führt ebenfalls zu solchen Fehlentwicklungen. Als Beispiel sei hier auf die zunehmende Praxis von Behörden und öffentlichen Dienststellen hingewiesen, Leistungen nur nach einem öffentlichen Bieterverfahren zu vergeben. Die fachlichen Anforderun-

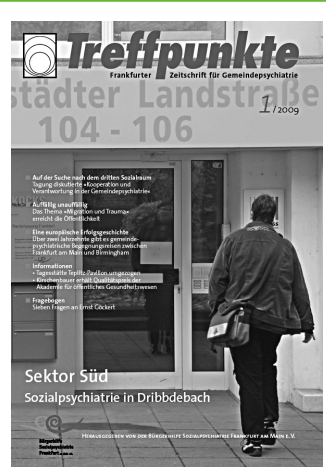

Die Sache mit den Sektoren ...

In Frankfurt am Main ist das Stadtgebiet bei den Hilfen für psychisch kranke Menschen in vier Versorgungsgebiete aufgeteilt. Mit den Sektoren Nord, Ost, West und Süd wurden nebeneinanderliegende Stadtteile zusammengefasst, um für die dort lebenden Menschen das gemeindepsychiatrische Hilfeangebot zu organisieren. Verantwortlich für einen Sektor ist jeweils eine Klinik sowie ein Trägerverein, der sich vorwiegend um die außerstationäre Versorgung mit sozialpsychiatrischen Angeboten kümmern soll. Zusätzlich gibt es für das gesamte Stadtgebiet den Bamberger Hof und das Internationale Fami- gen an die Leistungserbringer, die vorzuhaltenden Strukturen usw. sind dort häufig detailversessen beschrieben, jedes nur denkbare Zertifikat muss beglaubigt vorgelegt werden, Prozessund Strukturqualitäten sind bis auf die Stelle hinter dem Komma benannt. Und dann wird aufgrund des Vergaberechtes der Auftrag dreimal hintereinander an verschiedene Träger vergeben - und damit die Strukturen vor Ort zerstört oder zumindest nachhaltig durcheinandergebracht. Auch mit der Folge, dass die direkt betroffenen Menschen und die Kooperationspartner dieser Dienste fundamental verunsichert werden. Die Leistungen des Dienstes werden weniger nachgefragt, aufgrund der nachlassenden Nachfrage wird die Leistung insgesamt billiger: Öffentliche Mittel werden eingespart, eine wichtige Vorgabe wurde durch diese Art der Steuerung erreicht.

Die Verfahrensweise zur Vergabe eines Auftrages über die Erbringung einer sozialen Dienstleistung entscheidet über die langfristige Wirksamkeit und Akzeptanz dieser Leistung. Gleiches gilt übrigens für komplizierte Antrags- oder Bewilligungsverfahren im Einzelfall.

lienzentrum, die sich vor allem psychisch erkrankten Migrantinnen und Migranten widmen. Alle Einrichtungen zusammen verfügen über rund 460 Betten in Krankenhäusern, etwa 530 Arbeitsplätze in Werkstätten für psychisch kranke Menschen, nahezu 700 Wohnplätze in Wohnheimen und im Betreuten Wohnen, fünf Tagesstätten sowie Beratungsdienste und Begegnungsstätten. Koordiniert werden diese unterschiedlichen Hilfeeinrichtungen hauptsächlich durch die Abteilung Psychiatrie des Stadtgesundheitsamtes. Alle drei Partner - Kliniken, Trägervereine, Stadtgesundheitsamt - bilden den Gemeindepsychiatrischen Verbund. Hintergrund dieses Konzeptes aus dem Jahre 1986 war die Überzeugung, das Hilfesystem sollte nicht für die gesamten Einwohnerinnen und Einwohner der Stadt zentral geplant, sondern am besten für jeweils etwa 150.000 Menschen vor Ort organisiert werden.

Quelle: Bürgerhilfe Sozialpsychiatrie Frankfurt am Main e. V. (Hg.): Treffpunkte. Frankfurter Zeitschrift für Gemeindepsychiatrie. Heft 1/2009.

http://www.bsf-frankfurt.de/zeitschrift treffpunkte 